\title{
Regdanvimab: First Approval
}

\author{
Yahiya Y. Syed ${ }^{1}$
}

Published online: 1 November 2021

(c) Springer Nature Switzerland AG 2021, corrected publication 2021

\begin{abstract}
Regdanvimab (Regkirona ${ }^{\mathrm{TM}}$ ) is a recombinant human monoclonal antibody targeted against the severe acute respiratory syndrome coronavirus 2. It is being developed by Celltrion Inc. for the treatment of coronavirus disease 2019 (COVID-19). In September 2021, regdanvimab received full approval in South Korea for the treatment of COVID-19 in elderly patients aged $>50$ years with at least one underlying medical condition (obesity, cardiovascular disease, chronic lung disease, diabetes, chronic kidney disease, chronic liver disease, and patients on immunosuppressive agents) and mild symptoms of COVID-19 and in adult patients with moderate symptoms of COVID-19. This article summarizes the milestones in the development of regdanvimab leading to this first approval for COVID-19.
\end{abstract}

\section{Digital Features for this AdisInsight Report can be found at https://doi.org/10.6084/m9.figshare.16786057.}

\section{Regdanvimab (Regkirona ${ }^{\mathrm{TM}}$ ): Key Points}

A recombinant human monoclonal antibodybeing developed by Celltrion Inc. for the treatment of coronavirus disease 2019 (COVID-19).

Received its first approval on 17 September 2021 in South Korea.

Approved for use in COVID-19.

\section{Introduction}

Regdanvimab (Regkirona ${ }^{\mathrm{TM}}$ ) is a recombinant human monoclonal immunoglobulin G1 antibody targeted against the severe acute respiratory syndrome coronavirus 2

This profile has been extracted and modified from the AdisInsight database. AdisInsight tracks drug development worldwide through the entire development process, from discovery, through preclinical and clinical studies to market launch and beyond.

Yahiya Y. Syed

dru@adis.com

1 Springer Nature, Private Bag 65901, Mairangi Bay, Auckland 0754, New Zealand
(SARS-CoV-2), the causative virus of coronavirus disease 2019 (COVID-19). It is being developed by Celltrion Inc. for the treatment of COVID-19. In patients with confirmed COVID-19 and mild symptoms, early treatment can reduce the severity of the disease and associated hospitalization or ICU admittance [1]. Regdanvimab neutralizes SARS-CoV-2 by binding to the receptor binding domain (RBD) of the virus' spike protein [2]. The blockade of spike protein interaction with the angiotensin-converting enzyme 2 (ACE2) receptor prevents subsequent viral entry into human cells and viral replication [2]. The antibody retains antiviral activity against newer SARS-CoV-2 variants, which are associated with increased transmissibility, immune evasion and mortality (Sect. 2.1). Regdanvimab was identified through screening of an antibody library constructed from peripheral blood mononuclear cells of a Korean convalescent patient and selecting those with the highest affinity to the RBD [1,2].

Regdanvimab received its first full approval on 17 September 2021 in South Korea for the treatment of COVID19 in elderly patients aged $>50$ years with at least one underlying medical condition (obesity, cardiovascular disease, chronic lung disease, diabetes, chronic kidney disease, chronic liver disease, and patients on immunosuppressive agents) and mild symptoms of COVID-19 and in adult patients with moderate symptoms of COVID-19 [3, 4]. The recommended dosage is $40 \mathrm{mg} / \mathrm{kg}$, administered as a single 60-min intravenous (IV) infusion [3, 4].

In October 2021, a marketing authorization application for regdanvimab was submitted to the European Medicines Agency for the treatment of adults with COVID-19 who do not 


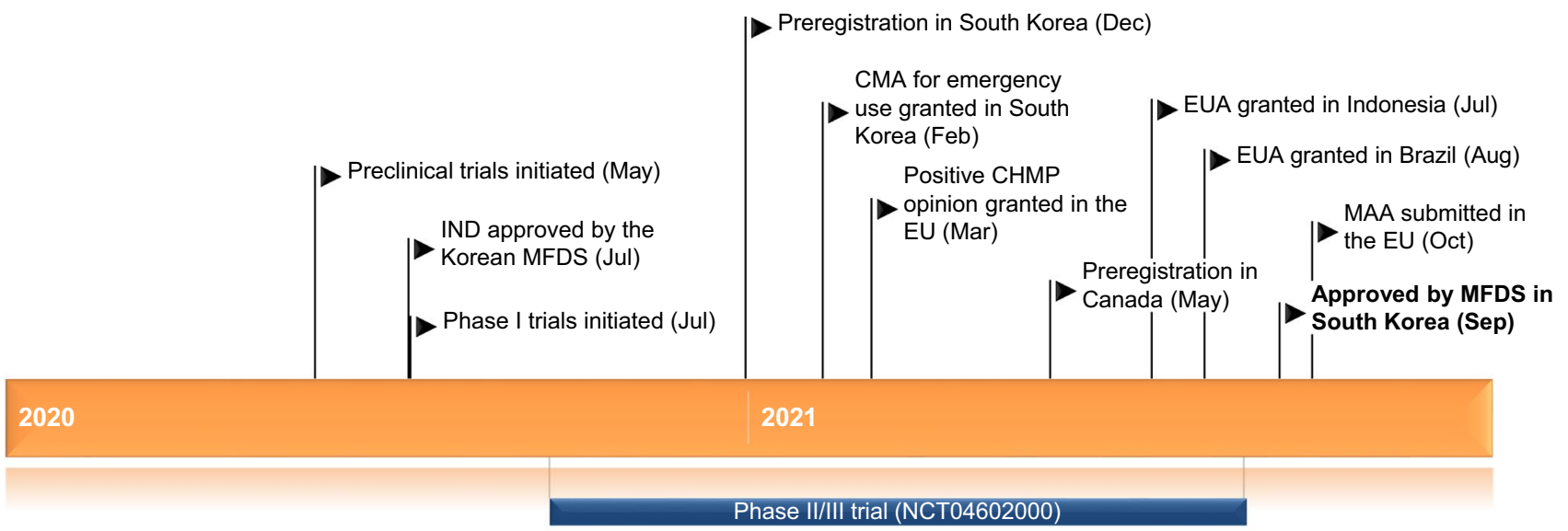

Key milestones in the development of regdanvimab. CHMP Committee for Medicinal Products for Human Use, CMA conditional marketing authorization, EUA emergency use authorization, IND investigational new drug application, MAA marketing authorization application, $M F D S$ Ministry of Food and Drug Safety

require supplemental oxygen therapy and who are at increased risk of progressing to severe COVID $19[5,6]$. The agency commenced a rolling review of regdanvimab in February 2021 [7] and concluded in March 2021 that the antibody can be used for the proposed indication [8]. Regdanvimab has been filed for approval in Canada for the treatment of COVID-19 [9] and is eligible for consideration by Australia's Therapeutic Goods Administration for the treatment of mild-to-moderate COVID-19 [10].

Celltrion is also developing a neutralising antibody cocktail with regdanvimab for COVID-19 [11]. In addition, a nebulised form of regdanvimab is being evaluated (in collaboration with Inhalon Biopharma) in a phase I study in healthy volunteers [12].

\subsection{Company Agreements}

In July 2021, Inhalon Biopharma and Celltrion entered in an agreement to develop a nebulised form of regdanvimab for the treatment of COVID-19, using Inhalon's muco-trapping antibody platform [13]. The formulation is expected to work by directly trapping the virus in airway mucus which will prevent the local spread of the infection and eliminate the virus from the lungs through the body's natural ability to clear mucus [13].

\section{Scientific Summary}

\subsection{Pharmacodynamics}

Regdanvimab decreased viral load in the upper and lower respiratory tracts in animal models of SARSCoV-2 infection [2]. The antibody showed antiviral activity against newly emerging variants of SARSCoV-2 (Gamma, Delta and its associated variants [14] and 501Y.V2 or B.1.351 [15]). In vitro, regdanvimab was able to neutralize these variants, albeit showing reduced binding affinity to their RBD. In transgenic mouse models inoculated with these variants, regdanvimab at clinically relevant dosages improved survival rate, reduced weight loss and decreased viral load in the upper and lower respiratory tracts $[14,15]$.

\subsection{Pharmacokinetics}

In a phase I trial (NCT04593641) in patients with mild SARS-CoV-2 infection, regdanvimab exhibited doseproportional pharmacokinetics over a dose range of $20-80 \mathrm{mg} / \mathrm{kg}$, with maximum plasma concentration $\left(\mathrm{C}_{\max }\right)$ increasing in a greater than dose-proportional manner [16]. The median time to $\mathrm{C}_{\max }$ was $2.5 \mathrm{~h}$; regdanvimab remained detectable in blood for up to 14 days [16].

In a part 1 of a phase II/III trial (NCT04602000) (Sect. 2.3.2) in patients with mild-to-moderate SARSCoV-2 infection, regdanvimab pharmacokinetics following a single $90-\mathrm{min}$ IV infusion was characterized by a low clearance $(0.227 \mathrm{~mL} / \mathrm{kg}$ for $40 \mathrm{mg} / \mathrm{kg})$, small volume of distribution $(87.2 \mathrm{~mL} / \mathrm{kg}$ for $40 \mathrm{mg} / \mathrm{kg})$ and a terminal half-life of 12 days [1, 17]. Regdanvimab is likely to be eliminated through normal immunoglobulin degradation pathways and the clearance is not expected to be affected by renal or hepatic impairment [1]. Thus, regdanvimab has no or minimal drug-drug interaction potential [1]. 


\begin{tabular}{|c|c|}
\hline Alternative names & CT-P59; Regkirona \\
\hline Class & Antivirals; Monoclonal antibodies \\
\hline Mechanism of action & Virus internalisation inhibitors \\
\hline Route of administration & Intravenous infusion \\
\hline Pharmacodynamics & $\begin{array}{l}\text { Neutralizes severe acute respiratory syndrome coronavirus } 2 \text { by binding to the receptor binding domain of the } \\
\text { virus' spike protein; decreases viral load in upper and lower respiratory tracts in animal models }\end{array}$ \\
\hline Pharmacokinetics & $\begin{array}{l}\text { Dose-proportional pharmacokinetics over a dose range of } 20-80 \mathrm{mg} / \mathrm{kg} \text {; median time to maximum plasma con- } \\
\text { centration } 2.5 \mathrm{~h} \text {; low clearance }(0.227 \mathrm{~mL} / \mathrm{kg} \text { for } 40 \mathrm{mg} / \mathrm{kg}) \text {; small volume of distribution }(87.2 \mathrm{~mL} / \mathrm{kg} \text { for } 40 \\
\mathrm{mg} / \mathrm{kg}) \text {; terminal half-life } 12 \text { days; eliminated through normal immunoglobulin degradation pathways }\end{array}$ \\
\hline Most frequent adverse events & Hypertriglyceridemia \\
\hline \multicolumn{2}{|l|}{ ATC codes } \\
\hline WHO ATC code & J05A-X (Other antivirals) \\
\hline EphMRA ATC code & J5B (Antivirals, excluding anti-HIV products) \\
\hline CAS Registry Number & $2444308-95-4$ \\
\hline
\end{tabular}

\subsection{Therapeutic Trials}

\subsubsection{Phase ITrial}

Regdanvimab demonstrated promising antiviral activity in adult patients with mild SARS-CoV-2 infection in a randomized, double-blind, placebo-controlled, single ascending dose (20, 40 and $80 \mathrm{mg} / \mathrm{kg}$ ) phase I trial (NCT04593641; $n=18$ ) [16]. The mean reduction from baseline in viral titers in nasopharyngeal swabs up to day 14 was generally greater with regdanvimab versus placebo. In patients with high titer levels at baseline $\left(10^{5}\right.$ copies $\left./ \mathrm{mL}\right)$, viral shedding was more rapid with regdanvimab versus placebo. All patients, except one placebo recipient, had clinical recovery from COVID19 by day 14 and the mean time to recovery was 3.39 days with regdanvimab (all doses combined) and 5.25 days with placebo [16].

\subsubsection{Phase II/III Trial}

The efficacy of regdanvimab in the treatment of mild-to-moderate COVID-19 was evaluated in a randomized, double-blind, placebo-controlled, multinational phase II/III trial (NCT04602000), which was divided in two parts: part 1 (phase II) and part 2 (phase III). Currently, part 1 results are available and regdanvimab was effective in this part [17]. Eligibility criteria included: age $\geq 18$ years; SARS-CoV-2 infection confirmed by quantitative reverse transcription polymerase chain reaction (RT-qPCR); > 94\% oxygen saturation on room air and not requiring supplemental oxygen; and, onset of symptoms (feverishness, cough, shortness of breath, sore throat, body/muscle pain, nausea, fatigue, headache, chills, nasal congestion, loss of taste or smell, or diarrhoea) within 7 days prior to study drug administration. Patients were randomized to a single dose of regdanvimab 40 or $80 \mathrm{mg} / \mathrm{kg}$, or placebo, administered as a 90-min IV infusion. The intent-to-treat (ITT) population included 105 and 111 patients in the regdanvimab 40 and $80 \mathrm{mg} / \mathrm{kg}$ groups, and 111 in the placebo group. Baseline demographic and disease characteristics were generally similar between the groups. In the ITT population, $87.5 \%$ of patients were white, $12.5 \%$ were Asian, $49.2 \%$ were female, median age was 51.0 years and $57.8 \%$ of patients had moderate disease. Efficacy was analysed in the ITT infected population, defined as all randomly assigned patients with confirmed SARSCoV-2 infection assessed by pre-infusion RT-qPCR result on day 1 and receiving a partial or complete dose of study drug (101 and 103 in the regdanvimab 40 and $80 \mathrm{mg} / \mathrm{kg}$ groups, and 103 in the placebo group) [17].

The median time to conversion to negative RT-qPCR (threshold $<2.33 \log _{10}$ copies/mL) up to day 28 (coprimary endpoint) in regdanvimab 40 and $80 \mathrm{mg} / \mathrm{kg}$ was 12.75 and 11.89 days versus 12.94 days with placebo; the improvement rate ratios $(95 \%$ CI) versus placebo were $1.346(1.001-1.810 ; p=0.048)$ and $1.215(0.90-1.63 ; p=0.198)$ for the 40 and $80 \mathrm{mg} / \mathrm{kg}$ groups, and $1.275(0.99-1.65 ; p=0.063)$ for the combined regdanvimab groups [17]. In a post hoc analysis, when a $<3.0 \log _{10}$ copies/ $\mathrm{mL}$ threshold was applied, the median time to conversion to negative RT-qPCR up to day 28 improved further: 5.94, 6.08 and 5.96 days with regdanvimab $40 \mathrm{mg} / \mathrm{kg}, 80 \mathrm{mg} / \mathrm{kg}$ and combined regdanvimab groups, respectively, versus 8.92 days with placebo; the corresponding improvement rate ratios $(95 \% \mathrm{CI})$ versus placebo were: 1.599 (1.185-2.158), 1.317 (0.976-1.777) and 1.451 (1.120-1.882). Furthermore, regdanvimab recipients had a $3 \log _{10}$ copies/mL reduction in viral load between baseline and day 7 , whereas a similar reduction was not seen in placebo recipients until day 10 . The proportion of patients achieving conversion to negative RT-qPCR up to day 28 was $92.1 \%$ and $87.4 \%$ in the regdanvimab 40 and $80 \mathrm{mg} / \mathrm{kg}$ groups, compared with $83.5 \%$ in the placebo group [17]. 


\begin{tabular}{llllll} 
Drug & Indication & Phase & Status & Location(s) & Identifier \\
\hline Regdanvimab & COVID-19 infections & II/III & Completed & Multinational & NCT04602000, EudraCT2020-003369-20, CT-P59 3.2 \\
Regdanvimab & COVID-19 infections & I & Completed & South Korea, Romania & NCT04593641, EudraCT2020-003165-19, CT-P59 1.2 \\
Regdanvimab & COVID-19 infections & I & Completed & South Korea & NCT04525079, EudraCT2020-003065-19, CT-P59 1.1 \\
\hline
\end{tabular}

The median time to clinical recovery up to day 14 was significantly ( $p \leq 0.039$ ) shorter for regdanvimab 40 and $80 \mathrm{mg} /$ $\mathrm{kg}$ versus placebo (5.35 and 6.23 days vs 8.77 days) [coprimary endpoint] [17]. Up to day $28,87.4 \%$ and $85.9 \%$ of patients in the regdanvimab 40 and $80 \mathrm{mg} / \mathrm{kg}$ groups, compared with $71.4 \%$ in the placebo group, achieved clinical recovery. The proportion of patients requiring hospitalization or oxygen therapy up to day 28 was $4.0 \%$ and $4.9 \%$ in the regdanvimab 40 and $80 \mathrm{mg} / \mathrm{kg}$ groups, compared with $8.7 \%$ in the placebo group; a similar trend was observed in patients with moderate infection $(6.5 \%$ and $7.9 \%$ vs $15.8 \%)$ and those with moderate infection and aged $\geq 50$ years ( $7.5 \%$ and $10.0 \%$ vs $23.7 \%$ ) [17].

Preliminary positive topline results from the phase III part $(n=1315)$ of the NCT04602000 trial have been reported by Celltrion. Regdanvimab significantly $(p<0.0001)$ reduced the risk of hospitalisation or death by $72 \%$ versus placebo in patients with mild-to-moderate COVID-19 symptoms who were considered at high risk of progressing to severe COVID-19 up to day 28 (3.1 vs. $11.1 \%$; primary endpoint). The trial also met other key secondary endpoints [18].

\subsubsection{Supportive Studies}

A Cochrane Database systematic review revealed that treatment with regdanvimab (solely based on NCT04602000 data) may decrease the risk of hospital admissions or death in outpatients with mild-to-moderate COVID-19 versus placebo [risk ratios (95\% CIs) $0.45(0.14-1.42)$ for $40 \mathrm{mg} /$ $\mathrm{kg} ; 0.56(0.19-1.60)$ for $80 \mathrm{mg} / \mathrm{kg}$ [ [19].

In a single-centre, retrospective, propensity score-matched (PSM) cohort study of patients with high-risk mild or moderate COVID-19, regdanvimab treatment was associated with a significant $(p<0.001)$ reduction in the proportion of patients reaching the composite of death or disease aggravation versus no regdanvimab treatment $(5.0 \%$ vs $21.5 \% ; n=377$ in each group; primary endpoint) [20]. The odds ratios $(95 \% \mathrm{CI})$ were $0.194(0.112-0.320)$ and $0.169(0.095-0.287)$ in univariate and multivariable-adjusted analyses, respectively $(p<0.001$ for both). Significantly $(p<0.001)$ fewer patients in the regdanvimab than in the no regdanvimab group were treated with remdesivir (2.7 vs 16.4\%), azithromycin (5.6 vs $62.3 \%$ ), lopinavir/ritonavir ( 0 vs $15.6 \%$ ) or corticosteroids (23.9 vs $47.7 \%$ ). Increased age, male gender, higher body mass index and concurrent pneumonia were associated with increased odds of reaching the primary endpoint. Results for the overall cohort ( $n=377$ for regdanvimab and 520 for no regdanvimab) were generally similar to those for the PSM cohort [20].

\subsection{Adverse Events}

In the phase II/III trial (NCT04602000), the overall incidence of treatment-emergent adverse events (TEAEs) was similar between the treatment groups (29.5\% and $24.5 \%$ with regdanvimab 40 and $80 \mathrm{mg} / \mathrm{kg}$ vs $30.9 \%$ with placebo) [17]. Treatment-related TEAEs occurred in $6.7 \%$ and $4.5 \%$ versus $4.5 \%$ of patients in the respective groups and the most common was hypertriglyceridemia (2.9\% with regdanvimab $40 \mathrm{mg} / \mathrm{kg}$ vs $1.8 \%$ with placebo). Most TEAEs were of grade 1 or 2 severity. Grade 3 TEAEs occurred in $4.8 \%$ and $3.6 \%$ of patients in the regdanvimab 40 and $80 \mathrm{mg} / \mathrm{kg}$ groups and $1.8 \%$ of patients in the placebo group, including one treatmentrelated event in the regdanvimab $40 \mathrm{mg} / \mathrm{kg}$ group. There were no grade 4 or 5 TEAEs up to day 28 , no serious TEAEs or TEAEs leading to treatment discontinuation or deaths. The incidence of infusion-related reactions, an adverse event (AE) of special interest, was low (1\% and $0 \%$ with regdanvimab 40 and $80 \mathrm{mg} / \mathrm{kg}$ vs $1.8 \%$ with placebo). The incidence of antidrug antibodies (ADAs) through day 28 was $0 \%$ and $2.7 \%$ versus $4.5 \%$ [17].

In the phase I trial (NCT04593641), 16 AEs were reported in 10 regdanvimab recipients and none were treatment related [16]. Grade 3 AEs occurred in two regdanvimab recipients (hepatocellular injury and increased ALT) and one placebo recipient (COVID-19 pneumonia). There were no infusion-related reactions, serious AEs or AEs leading to treatment discontinuation. Regdanvimab was not associated with any clinically meaningful changes in vital signs, hypersensitivity, ECG, radiography, physical examination or SARS-CoV-2 infection-related signs and symptoms. ADAs were not detected in any patient [16].

The Cochrane Database systematic review estimated that regdanvimab treatment may increase the risk of developing grades 3-4 AEs versus placebo [risk ratios (95\% CIs) 2.62 (0.52-13.12) for $40 \mathrm{mg} / \mathrm{kg}$; $2.00(0.37-10.70)$ for $80 \mathrm{mg} / \mathrm{kg}]$ [19].

In the PSM cohort study, regdanvimab treatment was associated with significantly ( $p<0.001$ vs no regdanvimab 
treatment) lower incidences of white blood cells increased $\left(>10 \times 10^{3} / \mu \mathrm{L} ; 5.0\right.$ vs $\left.11.9 \%\right)$ or decreased $\left(<4 \times 10^{3} /\right.$ $\mu \mathrm{L} ; 7.7$ vs $14.9 \%)$, decreased platelet counts $\left(<130 \times 10^{3} /\right.$ $\mu \mathrm{L} ; 2.4$ vs $5.8 \%$ ) and decreased absolute lymphocyte count $(<800 / \mu \mathrm{L} ; 2.9$ vs $11.9 \%)$ [20]. The two groups did not differ significantly for other investigated adverse reactions (renal and liver toxicity, gastrointestinal toxicity, fever or systemic pain, hypersensitivity, haemoglobin, absolute neutrophil count, and serum sodium and potassium abnormalities) [20].

\subsection{Ongoing Clinical Trials}

A post-marketing surveillance study of regdanvimab (CTP59 4.1) is ongoing.

\section{Current Status}

Regdanvimab received its first full approval on 17 September 2021 for the treatment of COVID-19 in South Korea [3].

Supplementary Information The online version contains supplementary material available at https://doi.org/10.1007/s40265-021-01626-7.

\section{Declarations}

Funding The preparation of this review was not supported by any external funding.

Authorship and Conflict of interest During the peer review process, Celltrion Healtcare Co., Ltd, was offered an opportunity to comment on the article. Changes resulting from any comments received were made by the authors on the basis of scientific completeness and accuracy. Yahiya Y. Syed is a salaried employee of Adis International Ltd/Springer Nature, and declares no relevant conflicts of interest. All authors contributed to the review and are responsible for the article content.

Ethics approval, Consent to participate, Consent to publish, Availability of data and material, Code availability Not applicable.

\section{References}

1. European Medicines Agency. Celltrion use of regdanvimab for the treatment of COVID-19: assessment report. 2021. https://www. ema.europa.eu/en/documents/referral/regdanvimab-treatmentcovid-19-celltrion-covid-19-article-53-procedure-assessmentreport_en.pdf. Accessed 1 Oct 2021.

2. Kim C, Ryu DK, Lee J, et al. A therapeutic neutralizing antibody targeting receptor binding domain of SARS-CoV-2 spike protein. Nat Commun. 2021;12(1):288.

3. Celltrion. Celltrions monoclonal antibody treatment for COVID19, regdanvimab (CT-P59) becomes the first authorized COVID19 treatment approved from the Korean Ministry of Food and Drug Safety (MFDS) [media release]. 18 Sep 2021. http://www. celltrionhealthcare.com.

4. Ministry of Food and Drug Safety. Regdanivmab full Korean approval. 2021. https://www.mfds.go.kr/brd/m_99/view.do?seq= 45778. Accessed 11 Oct 2021.
5. European Medicines Agency. EMA receives application for marketing authorisation for Regkirona (regdanvimab) for treating patients with COVID-19. 2021. https://www.ema.europa.eu/en/ news/ema-receives-application-marketing-authorisation-regki rona-regdanvimab-treating-patients-covid-19. Accessed 11 Oct 2021.

6. European Medicines Agency. Conditions of use, conditions for distribution and patients targeted and conditions for safety monitoring addressed to member states for unauthorised product Regkirona (regdanvimab). 2021. https://www.ema.europa. eu/en/documents/referral/celltrion-use-regdanvimab-treatmentcovid-19-article-53-procedure-conditions-use-conditions_en.pdf. Accessed 22 Sep 2021.

7. European Medicines Agency. EMA starts rolling review of Celltrion antibody regdanvimab for COVID-19 [media release]. 24 Feb 2021. http://www.ema.europa.eu.

8. European Medicines Agency. EMA issues advice on use of regdanvimab for treating COVID-19. 2021. https://www.ema.europa.eu/ en/news/ema-issues-advice-use-regdanvimab-treating-covid-19. Accessed 11 Oct 2021.

9. Celltrion. Celltrion Healthcare announces acceptance and priority review by Health Canada of New Drug Submission for its monoclonal antibody treatment for COVID-19, regdanvimab (CT-P59) [media release]. 2 Aug 2021. http://www.celltrionhealthcare.com.

10. Therapeutic Goods Administration. TGA grants provisional determination to Celltrion Healthcare Australia Pty Ltd's antiviral COVID-19 treatment regdanvimab [media release]. 23 Aug 2021. www.tga.gov.au.

11. Celltrion. Celltrion develops tailored neutralising antibody cocktail treatment with CT-P59 to tackle COVID-19 variant spread using its antibody development platform [media release]. $11 \mathrm{Feb}$ 2021. http://www.celltrionhealthcare.com.

12. Inhalon Biopharma. Inhalon doses first patient in phase 1 study of inhaled IN-006 to treat COVID-19 [media release]. 7 Oct 2021. https://www.inhalon.com/07october2021.

13. Inhalon Biopharma. Inhalon collaborates with Celltrion, Inc. to develop nebulized form of regdanvimab to treat COVID-19 patients at home [media release]. $22 \mathrm{Jul}$ 2021. http://www.inhalon.com.

14. Ryu DK, Kang B, Noh H, et al. The in vitro and in vivo efficacy of CT-P59 against gamma, delta and its associated variants of SARS-CoV-2. Biochem Biophys Res Commun. 2021;578:91-6.

15. Ryu DK, Song R, Kim M, et al. Therapeutic effect of CT-P59 against SARS-CoV-2 South African variant. Biochem Biophys Res Commun. 2021;566:135-40.

16. Kim JY, Jang YR, Hong JH, et al. Safety, virologic efficacy, and pharmacokinetics of CT-P59, a neutralizing monoclonal antibody against SARS-CoV-2 spike receptor-binding protein: two randomized, placebo-controlled, phase I studies in healthy individuals and patients with mild SARS-CoV-2 infection. Clin Ther. 2021. https://doi.org/10.1016/j.clinthera.2021.08.009.

17. Eom JS, Ison M, Streinu-Cercel A, et al. Efficacy and safety of CT-P59 plus standard of care: a phase $2 / 3$ randomized, doubleblind, placebo-controlled trial in outpatients with mild-to-moderate SARS-CoV-2 infection. Res Sq. 2021. https://doi.org/10. 21203/rs.3.rs-296518/v1.

18. Celltrion. Celltrion announces positive top-line results from global phase III trial of regdanvimab (CT-P59), an anti-COVID-19 monoclonal antibody treatment [media release]. 14 Jun 2021. http:// www.celltrionhealthcare.com.

19. Kreuzberger N, Hirsch C, Chai KL, et al. SARS-CoV-2-neutralising monoclonal antibodies for treatment of COVID-19. Cochrane Database Syst Rev. 2021. https://doi.org/10.1002/14651858. CD013825.pub2.

20. Heo J, Park S, Je NK, et al. Effectiveness and safety of regdanvimab in patients with mild-to-moderate COVID-19: a retrospective cohort study. Nat Portf. 2021. https://doi.org/10.21203/rs.3.rs-846378/v1. 